\title{
Changes of a mutualistic network over time: reanalysis over a 10 -year period
}

\author{
Cecilia Díaz-Castelazo, ${ }^{1}$ Paulo R. Guimarães, JR., ${ }^{2,3,4}$ Pedro Jordano, ${ }^{3}$ John N. Thompson, ${ }^{2}$ \\ Robert J. Marquis, ${ }^{5}$ and Víctor Rico-Gray ${ }^{1,6}$ \\ ${ }^{1}$ Departamento de Ecología Aplicada, Instituto de Ecología, A.C. Apdo. 63, Xalapa, Veracruz 91070 México \\ ${ }^{2}$ Department of Ecology and Evolutionary Biology, University of California, Santa Cruz, California 95064 USA \\ ${ }^{3}$ Integrative Ecology Group, Estación Biológica de Doñana, Consejo Superior de Investigaciones Científicas, \\ Avda. Américo Vespucio S/N, Isla de la Cartuja, E-41092 Sevilla, Spain \\ ${ }^{4}$ Departamento de Ecologia, Instituto de Biociências, Universidade de São Paulo, 05422-970, SP, Brazil \\ ${ }^{5}$ Department of Biology, University of Missouri-St. Louis, R223 Research Building, One University Boulevard, \\ St. Louis, Missouri 63121-4499 USA
}

\begin{abstract}
We analyzed the structure of a multispecific network of interacting ants and plants bearing extrafloral nectaries recorded in 1990 and again in 2000 in La Mancha, Veracruz, Mexico. We assessed the replicability of the number of interactions found among species and also whether there had been changes in the network structure associated with appearance of new ant and plant species during that 10-year period. Our results show that the nested topology of the network was similar between sampling dates, group dissimilarity increased, mean number of interactions for ant species increased, the frequency distribution of standardized degrees reached higher values for plant species, more ant species and fewer plant species constituted the core of the more recent network, and the presence of new ant and plant species increased while their contribution to nestedness remained the same. Generalist species (i.e., those with the most links or interactions) appeared to maintain the stability of the network because the new species incorporated into the communities were linked to this core of generalists. Camponotus planatus was the most extreme generalist ant species (the one with the most links) in both networks, followed by four other ant species; but other species changed either their position along the continuum of generalists relative to specialists or their presence or absence within the network. Even though new species moved into the area during the decade between the surveys, the overall network structure remained unmodified.
\end{abstract}

Key words: ant-plant interactions; Camponotus planatus; extrafloral nectary-bearing plants; generalist vs. specialist species; Mexico; mutualistic networks; temporal dynamics.

\section{INTRODUCTION}

Interspecific interactions evolve as a geographic mosaic, generating not only differences across space and time in traits and ecological outcomes, but also in the number of species involved (e.g., Thompson 2005). As these webs of interaction grow in the number of interacting species, they converge on different network structures that depend upon multiple factors including the nature of the interaction (Bascompte and Jordano 2007). Recent studies have shown that mutualistic networks exhibit complex but predictable network structures, frequently involving many species (Jordano et al. 2003, Bascompte and Jordano 2007). Multiple analyses have shown that mutualistic networks among free-living species are nested, such that generalist species interact with each other forming a central core of species, specialists interact with generalists, and interactions between specialists are usually absent. The overall

Manuscript received 10 October 2008; revised 4 May 2009; accepted 1 June 2009. Corresponding Editor: D. A. Holway.

${ }^{6}$ Corresponding author.

E-mail: victor.rico@inecol.edu.mx pattern is one of weak and asymmetric links among species (Bascompte et al. 2003, 2006, Bascompte and Jordano 2006, Guimarães et al. 2006, Vázquez et al. 2007). These network patterns may be explained by ecological processes and evolutionary history, but few data exist on their spatial and temporal stability.

One source of spatial and temporal variability is disruption caused by introduced or new species that are not normal or constant parts of local networks. New species can impose strong ecological and evolutionary effects on network structure, as has already been shown in analyses of plant-pollinator interactions (Memmot and Waser 2002, Olesen et al. 2002, Aizen et al. 2008, Olesen et al. 2008). The new mutualists sometimes become wellintegrated into pollination networks and have only a slight effect, if any, on the degree of connectance among species (i.e., the number of realized links; Bascompte and Jordano 2007) relative to the original network (Memmot and Waser 2002, Olesen et al. 2002). Other studies, however, have indicated that some new species can modify the structure of pollination networks (Simberloff and Von Holle 1999, Aizen et al. 2008). 
Once established, new species could increase in abundance and dominate an entire community through direct and indirect self-perpetuating mechanisms (Aizen et al. 2008). Moreover, given the possibility that a core of generalist species may drive the evolution of an entire network (Thompson 2005, Guimarães et al. 2007), it is important to assess how the presence of these new species may change networks in ways that alter the ecological structure of these interactions.

Here we explore temporal variation in mutualistic network structure, mediated by the presence of new species and changes in the positions of other local species within a network. We analyzed changes in antplant interactions mediated by the presence of extrafloral nectaries on plants. We first sampled this network between May 1989 and April 1991 (RicoGray 1993) in La Mancha, Veracruz, Mexico, and then again in the same habitats between October 1998 and September 2000 (Díaz-Castelazo et al. 2004). This sampling scheme allowed us to assess the replicability of this kind of community-wide description. Extrafloral nectary-mediated ant-plant mutualisms have been the focus of recent network analysis (Guimarães et al. 2006, 2007, Blüthgen et al. 2007; see also Fonseca and Ganade 1996), and are among the most temporally and spatially variable of mutualistic interactions that have been studied (Rico-Gray and Oliveira 2007). We also tested whether changes in the network over the past decade were associated with the presence of new species. Our definition of new ant species includes "invasive species," which are those that move into naturally disturbed or undisturbed habitats and outcompete native ant species; "tramp species," which are those closely associated with human activity and often nest in human structures; and introduced species (McGlynn 1999, Schultz and McGlynn 2000, Holway et al. 2002). New plant species include "ruderal plants," which are often associated with human disturbance (Moreno-Casasola 2006). For our purposes, any of these lifestyles was considered as a new species to the network. Furthermore, we considered a new species as one that had not been in the habitat long enough to be considered part of the evolutionary history of that community. However, there is also the possibility that these ant species were formerly present but have gone extinct in the past few decades, and have recently returned. Multiple changes other than the appearance of new species have undoubtedly occurred during the decade between the two censuses, but our comparison allows an analysis of where new species may fit within the network and how the community thus reacted to their presence.

\section{Materials and Methods}

\section{Study site}

Fieldwork was carried out at Centro de Investigaciones Costeras La Mancha (CICOLMA), located on the coast of the state of Veracruz, Mexico $\left(19^{\circ} 36^{\prime} \mathrm{N}\right.$, $96^{\circ} 22^{\prime} \mathrm{W}$; elevation $<100 \mathrm{~m}$ ). The climate is warm and subhumid; a rainy season occurs between June and September, total annual precipitation is $\sim 1500 \mathrm{~mm}$, and mean annual temperature is $22^{\circ}-26^{\circ} \mathrm{C}$. The major vegetation types in the study area are tropical deciduous forest, tropical dry forest, sand dune scrub, mangrove forest, freshwater marsh, and flooded deciduous forest (surrounding a freshwater lagoon; see Moreno-Casasola 2006). In most vegetation types at the study site, changes in the abundance of associations between ants and plants bearing extrafloral nectaries (EFNs) suggest that ant-plant interactions are strongly influenced by climatic conditions as a result of marked seasonality (DíazCastelazo et al. 2004, Rico-Gray and Oliveira 2007).

\section{Field observations and data collection}

Ant-plant interactions were sampled first between May 1989 and April 1991 (Rico-Gray 1993), and then again between October 1998 and September 2000 (DíazCastelazo et al. 2004). In the analyses below these are referred to, for simplicity, as Network 1990 and Network 2000, respectively. All temporal data were pooled for analyses.

Sampling and methods were the same for both studies. Biweekly field observations were conducted along six arbitrarily selected but representative $1-\mathrm{km}$ trails that sampled different vegetation associations: Trail 1, sand dune pioneer species; Trail 2, deciduous forest; Trail 3, deciduous forest-dry forest ecotone; Trail 4, dry forest and sand dune scrub; Trail 5, sand dune scrub; and Trail 6, sand dune-fresh water lagoon ecotone and mangrove forest. We recorded all occurrences of ants collecting liquids from plants. On each visit we noted ant species, plant species, and the food source or structure mediating the ant-plant interaction. Once an individual plant was marked as visited by ants, it was subsequently re-checked throughout the study. The food resource used by ants was extrafloral nectar, produced either by the surface of reproductive structures such as the spike, pedicel, bud, calyx, or fruit, or secreted by special structures on vegetative parts such as leaves, shoots, petioles, bracts, or stems (Fig. 1). Ants were considered to be feeding on nectar when they were immobile, with mouthparts in contact with nectarsecreting tissues, for periods of up to several minutes. Nectar-feeding ants often showed obviously distended gasters (see also Rico-Gray 1993).

\section{Metrics and data sets}

We analyzed changes in species composition and network structure after standardizing both surveys (e.g., night samples were eliminated from Network 2000). Structural features analyzed for each network included links, defined as the interaction between a plant species and an ant species mediated by a nectar secreting tissue, standardized degree, which is the number of links per species divided by the maximum number of links possible for the plants or the ants within that network, 

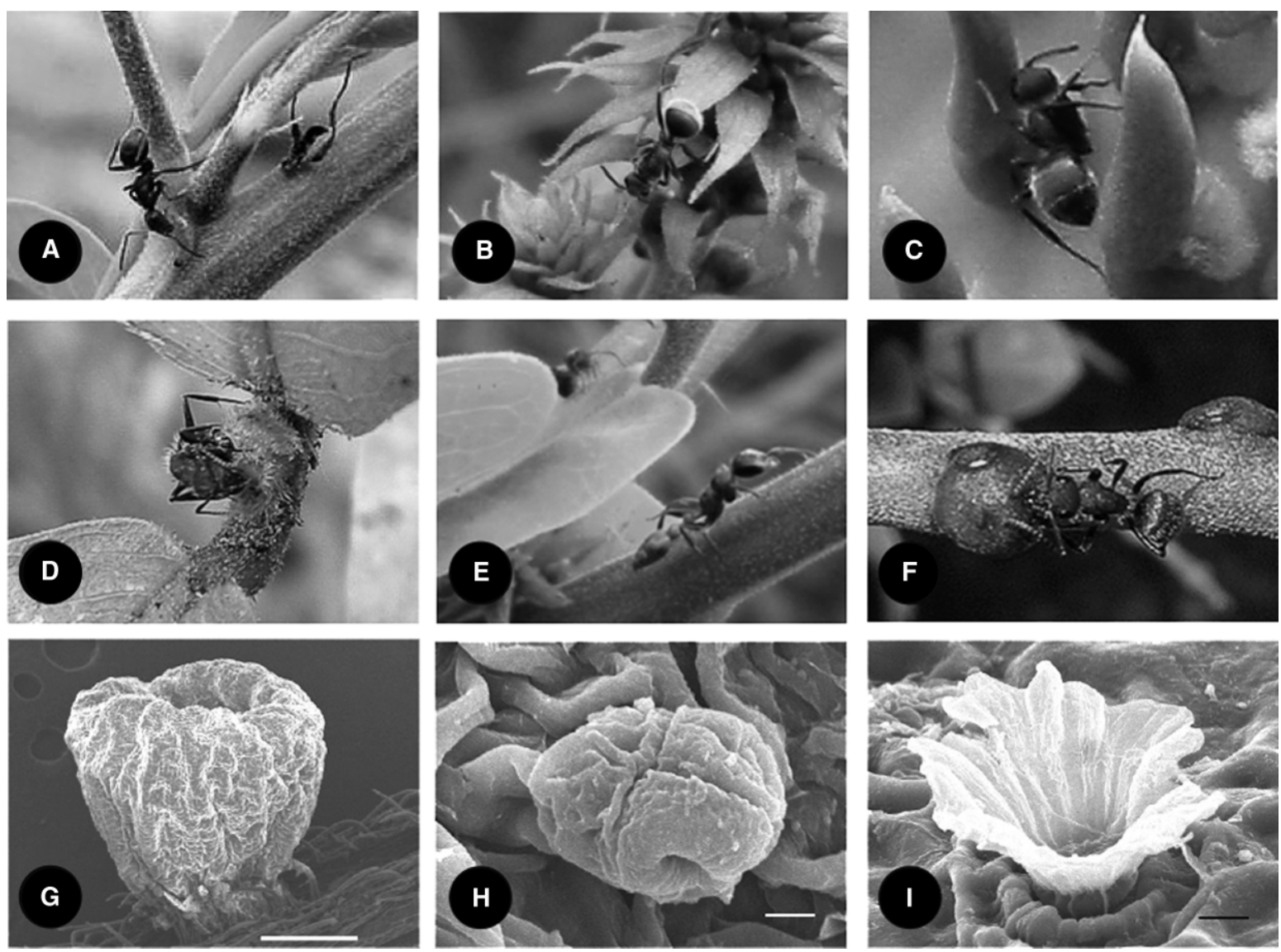

FIG. 1. Plant species bearing extrafloral nectaries at Centro de Investigaciones Costeras La Mancha, Mexico: (A) Crotalaria incana L., (B, E) Caesalpinia crista L., (C) Opuntia stricta Haworth, (D) Prestonia mexicana A. DC., and (F) Canavalia rosea (Swartz) DC. All ant foragers shown are Camponotus planatus Roger (the ant species with the highest number of links), except for the ant in panel (E), which is Pseudomyrmex gracilis (Fabricius). (G-I) Scanning electronic microscope images of extrafloral nectaries (modified from Díaz-Castelazo et al. [2005]): (G) cup-shaped elevated vascularized nectary of Chamaecrista chamaecristoides (Collad) I. \& B. stalked on the petiole of the compound leaf (scale bar $=200 \mu \mathrm{m}$ ); $(\mathrm{H})$ capitated nonvascularized secretory trichome on the calyx of Macroptilium atropurpureum (Sessé \& Mociño ex DC.) Urban (scale bar = 5 $\mu \mathrm{m}$ ); (I) scale-like nonvascularized secretory trichome on the underside of the leaf of Tabebuia rosea (Bertol.) DC. (scale bar $=5 \mu \mathrm{m}$ ).

and its cumulative distribution $P(k)$. We analyzed the mean degree values for plants and ants, which are the sum of the links for species in each set, ants or plants, divided by the number of species in the set. We also analyzed network connectance, defined as the proportion of realized links of the total possible in each network; $C=I /(P \times A)$, where $I$ is the total number of interactions recorded for the network, $P$ is plant species richness, and $A$ is ant species richness; nestedness, defined as a network pattern consisting of a core of reciprocal generalists accompanied by specialist species that interact almost exclusively with generalists (Atmar and Patterson 1993, Bascompte et al. 2003); and speciesspecific contributions to nestedness (Atmar and Patterson 1993). The latter allowed us to identify the proportion of idiosyncratic species (i.e., species that show patterns of interactions in a way that departs from a perfectly nested pattern), as well as nestedness contributions of new species among networks.
We also characterized a given species as part of the central core of species or, alternatively, as one of the peripheral species. We used categorical core-periphery analysis for bipartite networks (Borgatti and Everett 1999, Borgatti et al. 1999). In this analysis, core and periphery are identified by sorting ant and plant species in such a way that the connectance among core species is maximized while minimizing connectance among peripheral species. We recorded the proportion of new species in both core and periphery of the two networks. Finally, we computed dissimilarities within groups of species (plants and ants) and mean network dissimilarity, described by $d 1$ Ward Euclidean dissimilarity (de Nooy et al. 2005), in the association patterns of each group (plants and ants). A dendrogram of dissimilarity patterns for each group within each network was estimated. In this analysis, two ant species with a dissimilarity score of 0 (clustering at the same level in the dendrogram) visited exactly the same plant species, 
whereas a dissimilarity score of 1 indicated that no plant species was visited by the same two ant species (de Nooy et al. 2005).

\section{Data analysis and statistics}

Initial computations were done using Microsoft Excel. The cumulative distribution of standardized degrees, network graphs, and dissimilarity analyses were performed with Pajek (de Nooy et al. 2005; available online).$^{7}$ Analysis of nestedness, species contribution to nestedness, and determination of idiosyncratic species were performed with ANINHADO (Guimarães and Guimarães 2006; available online). ${ }^{8}$ We compared the observed degree of nestedness of each network with 1000 replicates generated by a null model to assess whether the degree of nestedness was higher than expected by random patterns of interaction. The metric used to characterize nestedness was the degree of nestedness (Bascompte et al. 2003), a metric based on the temperature of the matrix (Atmar and Patterson 1993). We used matrix temperature because this metric allowed us to quantify the contribution of each species to nestedness (Atmar and Patterson 1993, Selva and Fortuna 2007). The null model chosen for the analysis was CE (or null model 2; Bascompte et al. 2003), which implies that if the number of links per species is correlated with abundance, then the null model controls for important differences in species abundances and potential sampling bias (Guimarães et al. 2006). The $P$ value was defined as the probability of a null model replicate being equally or more nested than the observed networks. Analyses of species as core or peripheral components of the network were performed with UCINET 6 for Windows (Borgatti et al. 2002; Analytic Technologies, available online), ${ }^{9}$ which performs categorical core/periphery analysis for bipartite graphs (Borgatti and Everett 1999, Borgatti et al. 1999). Because the core-periphery analysis is based on stochastic-based optimization processes, we performed 20 runs for each network, obtaining the proportion of occurrences of a species within the core or the periphery for the entire set of runs. We investigated if any given species was more associated to either the core or periphery than expected by chance by estimating the probability that the observed proportion of occurrences for each species in the core (or periphery) could have been obtained by chance through a binomial distribution.

Tests for differences in proportions and distributions of species between networks were calculated using XLStat 2008 (Addinsoft, New York, New York, USA). Differences in the proportion of new species between networks were tested with a $Z$ test for comparing two proportions. Because the data were neither normally

\footnotetext{
${ }^{7}\langle$ http://vlado.fmf.uni-lj.si/pub/networks/pajek/〉

8 〈www.guimaraes.bio.br〉

${ }^{9}\langle$ http://www.analytictech.com/downloaduc6.htm $\rangle$
}

distributed nor normalized through transformations (e.g., arcsine transformation), comparisons of network parameters were carried out with Kolmogorov-Smirnov two-sample tests for comparing the distributions of two samples of continuous observations through the $D$ statistic (Sokal and Rohlf 1995). These comparisons between networks involved the cumulative distribution of standardized degree, species-specific contributions to nestedness, frequency distribution of links per species, nestedness contribution of new species, and cumulative distributions for standardized number of links of new species. The cumulative distribution of standardized number of links provided non-arbitrary cutoffs for considering species as generalists or specialists within each network (cutoffs were based on cumulative distributions). Comparing nestedness contributions and the standardized number of links of new species between each network allowed us to explore if the appearance of new species in the communities studied modified network structure and topology (see Guimarães et al. 2006, 2007, and Bascompte and Jordano 2007, for full details on metrics and statistical analyses).

\section{RESUlts}

\section{Standardized degree and its cumulative distribution}

Network 1990 included 50 plant species and 23 ant species in 159 associations, whereas Network 2000 included 40 plant species and 30 ant species in 208 associations (Fig. 2; plant and ant species are listed in Appendix A). Networks differed in the number of plant species, ant species, and associations, despite sharing 17 plant and 16 ant species (mean values in Table 1). Hence, the networks exhibited a very high species turnover. Standardized cumulative distribution of links per species differed significantly between years for both plant (Kolmogorov-Smirnov, $D=0.260, P<0.05, N_{1990}$ $\left.=50, N_{2000}=40\right)$ and ant species $(D=0.339, P<0.05$, $N_{1990}=23, N_{2000}=30$ ). The standardized average number of links for plants was larger for Network 2000 compared to Network 1990. In Network 1990 the standard degree and standard deviations for the number of links were smaller $(0.39 \pm 0.01$, mean \pm SD) compared to those recorded for the plants in Network $2000(0.57 \pm 0.14)$. The cumulative distribution of standardized number of links for ants was similar between networks (Network $1990=0.80 \pm 0.17$; Network $2000=0.802 \pm 0.17$ ). Thus after 10 years the network included more generalist species, but these changes in the average number of links were mainly caused by plants. Overall network connectance values (0.138 for Network 1990 and 0.173 for Network 2000) confirm these temporal differences (Table 1).

\section{Nestedness}

Nestedness values did not differ between networks ( $N$ $=0.999$ for both Network 1990 and Network 2000), both exhibiting a highly significant nested topology $(P<$ 0.001). Although not significant $\left(\chi^{2}=3.06, P=0.080\right)$, 

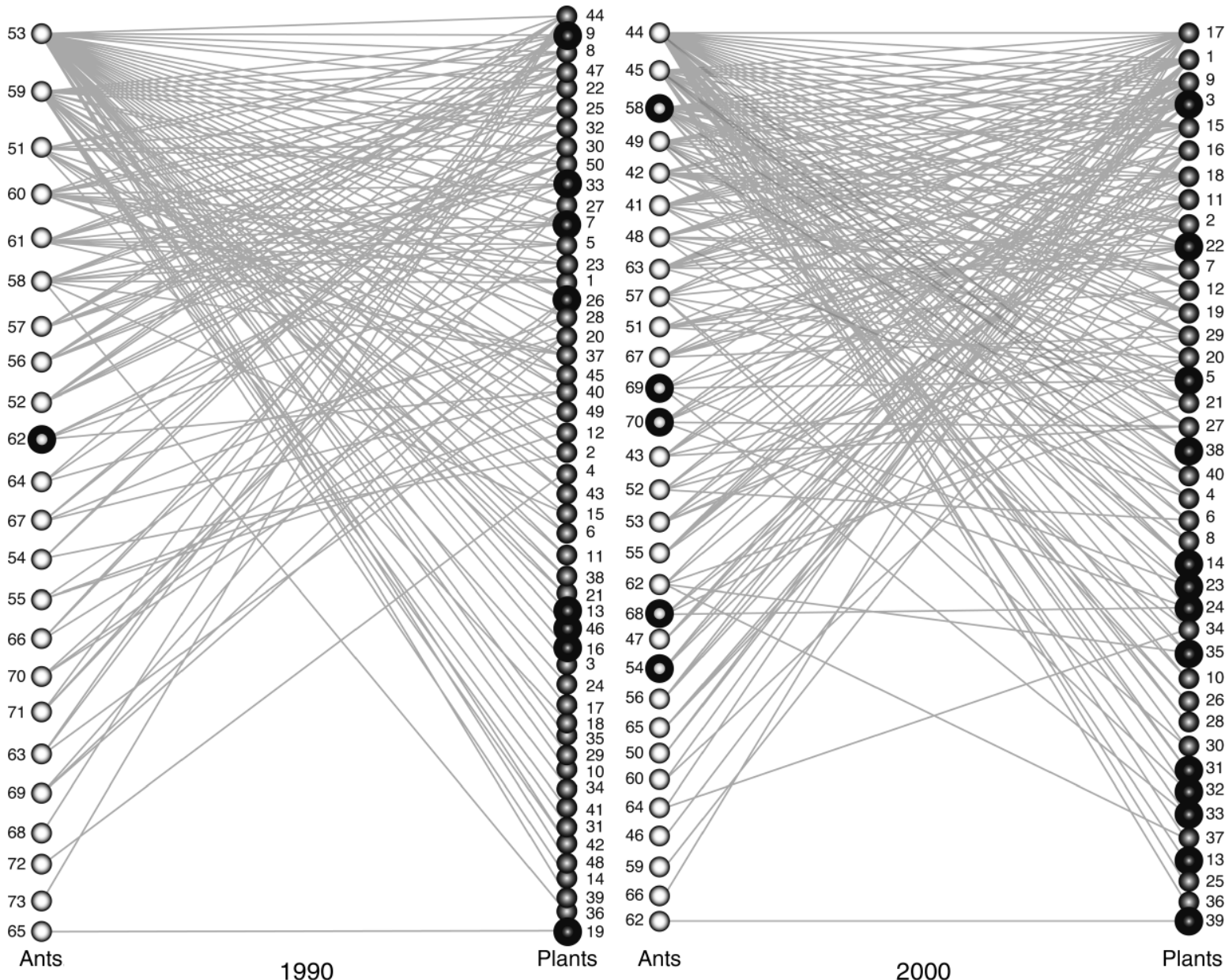

FIG. 2. Ant-plant interaction networks at Centro de Investigaciones Costeras La Mancha, Mexico, on the coast of Veracruz, Mexico, in 1990 and 2000. Circles represent species, and links indicate extrafloral nectar feeding associations among ants and plants. Network 1990: 23 ant species and 50 plant species. Network 2000: 30 ant species and 40 plant species (see Appendix A for species identities). Bolded vertices denote new ant and plant species.

there is an indication that plant species exhibited more nested patterns of interaction in Network 1990 than in Network 2000 (39 vs. 25 species, respectively), corresponding to $78 \%$ and $62.5 \%$ of the total species richness in each network. The percentage of idiosyncratic plant species did not differ significantly between networks (Network $1990=11$ species or $22 \%$, Network $2000=15$ species or $37.5 \% ; \chi^{2}=0.62, P=0.431$ ). The percentages of ant species that contributed to nestedness did not differ significantly between networks (14 and 18 species; $\left.\chi^{2}=0.50, P=0.479\right)$. The number of idiosyncratic species per network did not differ significantly $(1990=9$ species; $2000=12$ species; $\chi^{2}=0.43, P=0.412$ ).

\section{Changes in species distributions}

Camponotus planatus Roger was the most extreme generalist ant species (the one with the most links with plants) in both networks, interacting with 40 plant species in 1990 and 33 in 2000. Other important generalist ant species found in both networks were
Crematogaster brevispinosa Mayr, Camponotus mucronatus Emery, Azteca sp., Forelius analis André, and Dorymyrmex bicolor Wheeler. The rest of the species changed either in their position (generalist to specialist)

TABLE 1. Number of ant and plant species, mean number of links values, connectance, nestedness, and mean dissimilarities for the networks studied at Centro de Investigaciones Costeras La Mancha, Mexico.

\begin{tabular}{lcc}
\hline \hline \multicolumn{1}{c}{ Network metrics } & $\begin{array}{c}\text { Network } \\
1990\end{array}$ & $\begin{array}{c}\text { Network } \\
2000\end{array}$ \\
\hline Number of plant species & 50 & 40 \\
Number of ant species & 23 & 30 \\
Number of associations & 159 & 208 \\
Mean number $( \pm \mathrm{SE})$ of links & $6.9 \pm 0.3$ & $5.2 \pm 0.6$ \\
$\quad$ for plant species & $3.2 \pm 1.8$ & $6.9 \pm 1.3$ \\
Mean number $( \pm \mathrm{SE})$ of links & & \\
$\quad$ for ant species & 0.138 & 0.173 \\
Network connectance & 0.99 & 0.99 \\
Nestedness value $(N)$ & 0.128 & 0.243 \\
Mean dissimilarity for plants & 0.333 & 0.352 \\
Mean dissimilarity for ants & &
\end{tabular}


or in their presence/absence when the two networks were compared (Fig. 2). In Network 2000, the ant fauna was composed of more generalist species (11 species, or $15.7 \%$ of the total species, had 10 or more links with plants; four species, or $5.7 \%$ of the total species, had less than five links). Network 1990 had more specialist species. Six species $(8.2 \%)$ had 10 or more links with plants, and 18 species $(24.6 \%)$ had fewer than five links. Furthermore, Network 2000 had eight species (11.4\%) that interacted only with one species, either plant or ant, whereas Network 1990 had 22 (30.1\%) specialist species.

\section{Core/periphery distribution of species}

The proportion of species constituting the core and the periphery in each network did not change with time. However, the identities of species constituting the core and the periphery did change (see Appendix A for the list of species). In Network 1990 only two ant species (Camponotus planatus and Crematogaster brevispinosa) were part of the core in $95 \%$ or more runs $(P<0.0001)$, and 17 ant species (74\% of ant species in the network) were never included in the core (strictly peripheral, $P<$ 0.0001). For network 2000, $C$. planatus and $C$. brevispinosa were still core species $(>95 \%$ of runs, $P<$ 0.001). In addition, Camponotus mucronatus and Paratrechina longicornis (Cantreille) were also frequently associated with the core of the network ( $>95 \%$ of runs, $P<0.0001) ; 20$ ant species $(67 \%$ of ant species in the network) were strictly peripheral $(P<0.0001)$. For plants, 15 species in Network 1990 and eight species in Network 2000 were part of the core of species in $95 \%$ or more runs $(P<0.0001)$. Only one species in Network 1990 and four species in Network 2000 were strictly peripheral $(P<0.0001)$. In contrast to ant species, plant species found occasionally at the core of each network exhibited higher turnover, as 16 plant species were common to both networks (32\% of plant species in Network 1990 and 40\% in Network 2000), whereas only three species (Turnera ulmifolia L., Canavalia rosea (Swartz) DC, and Crotalaria incana L.; i.e., $18 \%$ of the total plant species common to both networks) were constant core members in both networks for $95 \%$ or more of runs $(P<0.0001)$.

The change in the species at the periphery of the networks was $78 \%$ for Network 1990 and $80 \%$ for Network 2000. When we analyzed changes in species composition at the periphery of both networks, we found that the change of plant species was $100 \%$. No peripheral plant species in Network 1990 remained at the periphery in Network 2000. Similarly, ant species at the periphery changed greatly, but five ant species remained peripheral in Network 2000.

\section{Dissimilarities in association patterns}

Niche overlap was higher for plants and ants in Network 1990 than in Network 2000 (Table 1). The analysis of dissimilarity dendrograms revealed differences for both ants and plants in the patterns of associations with partners (see Appendices B and C). The dissimilarity dendrogram for ants in Network 1990 showed four distinct clusters. Two generalist ant species (i.e., those with the most links) were the most dissimilar, followed by a three-species cluster with moderately high number of interactions and a cluster formed by three species with exactly the same intermediate number of interactions, and an ant species (Azteca sp.) that shared with them its association patterns with plants, despite having a high number of interactions. Thus generalists tended to interact with each other.

For plants, the dissimilarity dendrogram showed two clusters that were separate from the rest. These clusters included the plant species visited by most ant species. Many plant species exhibited zero dissimilarity since they shared visitation by only one ant species, and very often it was the same species. Within each of the two clusters that separate from the rest, the plant species included shared the same vegetation type: the bottom cluster (the most dissimilar) was composed of plant species from the freshwater marsh-deciduous forest ecotone and the cluster above was composed of plant species from the sand dune vegetation.

The most distant cluster in the dissimilarity dendrogram for the ants in Network 2000 grouped the ant species that visited the highest number of plant species: C. planatus (33 plant species), C. mucronatus (22 plant species), and $P$. longicornis (18 plant species). In contrast, the ant species with lower dissimilarities included the specialist species visiting very few plant species. Similarly, the most distant cluster in the dissimilarity dendrogram for the plants in Network 2000 grouped the species that were visited by most ant species: Cedrela odorata L. (17 ant species), Turnera ulmifolia (15 species), Cordia spinescens L. (15 species), Crotalaria incana (11 species), Callicarpa acuminata Kunth (11 species), Calopogonium caerulium (Benth.) Sauvalle (10 species), and Amphilophium paniculatum (L.) HBK (6 species).

\section{New ant and plant species}

The networks differed in the richness and number of interactions of new ant species (e.g., Solenopsis geminata (Fabricius), Wasmannia auropunctata Roger, Monomorium floricola Jerdon, and Tetramorium spinosum Pergande; Fig. 2, Table 2). All were present in Network 2000 but absent in Network 1990. Only Paratrechina longicornis was present in both networks (see Introduction), as a peripheral species with few interactions (visiting only four plant species) in Network 1990, and as a generalist species in Network 2000 (visiting 18 plant species). Furthermore, new plant species were better represented in Network 2000 (1990, eight species or $16 \%$ of plant species; 2000, 13 species or $32.5 \%$ of plant species; Fig. 2, Table 2).

The proportion of new species (whether plants or ants) in Network 1990 (0.123) was significantly smaller than that of Network $2000(0.257 ; Z=-2.048, P<$ 
TABle 2. Network attributes of new plant and ant species at Centro de Investigaciones Costeras La Mancha, Mexico.

\begin{tabular}{lll}
\hline \hline \multicolumn{1}{c}{ Network metrics } & Network 1990 & Network 2000 \\
\hline Number (percentage) of new plant species & $8(16.0 \%)$ & $13(32.5 \%)$ \\
Number (percentage) of new ant species & $1(4.3 \%)$ & $5(16.7 \%)$ \\
Proportion of new species & $0.123(9$ of 73$)$ & $0.257(18$ of 70$)$ \\
Nestedness contribution of new species & 0.093 & 0.090 \\
Proportion of new plant species with low number of links (below mean) & $0.875(7$ of 8) & $0.923(12$ of 13) \\
Proportion of new ant species with low number of links (below mean) & 1 (1 of 1) & $0.8(4$ of 5) \\
Association of new plants with alien ants (less than expected for both networks) & 1 association of & 11 associations of \\
& 658 chances & 65 chances \\
\hline
\end{tabular}

0.010, $N_{1990}=73, N_{2000}=70 ;$ Table 2). Nestedness contribution of new species did not change between networks $\left(D=0.444, P=0.172, N_{1990}=9, N_{2000}=18\right.$; i.e., new species in the networks contributed similarly to their nested pattern; Table 2). However, the presence of new species at the core or at the periphery of each network was different. For Network 1990, 25\% of new plant species occurred at the periphery, while for Network $200046 \%$ of new plant species occurred at the periphery, thus positioning the new species as nonkey components of the network. In contrast, $P$. longicornis was strictly peripheral in Network 1990, whereas 10 years later it occupied a key position, since it was a core component in $100 \%$ of runs.

\section{DiscusSION}

\section{The distribution of number of interactions per species and nestedness}

The number of interacting species and the structural positions of individual species within the networks differed ten years after the initial study. In the more recent census, the plant species bearing extrafloral nectaries exhibited a more species-rich community of ant species. However, both networks were highly nested, suggesting that the structure of the network was resilient to changes in species number and number of links, and to the arrival of new species. The most recent network (Network 2000) included more generalist species of plants and ants, and more interactions among them. In contrast, Network 1990 was characterized by a smaller number of idiosyncratic species and by more species of specialist plants and ants, mainly because one or two ant species visited many plant species and the rest of the ants tended to visit few plants, leading to great overlap for the plant community; a few ant species dominated the assemblage in Network 1990.

\section{Core/periphery distribution of species and dissimilarities}

A pattern emerging from the core-periphery analysis is that, with time, ants became more important as core components within the networks, while plants became less important. This pattern could be explained because (1) plant communities at the study site are subject to a recurrent successional process (e.g., sand dune movement, tropical storms, seasonal variations in the level of flood by sea and freshwater; Rico-Gray and Castro
1996, Moreno-Casasola 2006), and (2) ants have more links than their plant counterparts (based on our results). This suggests that in the scenario of recurrent natural plant succession and increasing transformation of plant communities caused by human activities at La Mancha (Moreno-Casasola 2006), generalist ant species rapidly switch food plant species as they change in abundance. Furthermore, plant succession leads to stronger stratification of ant assemblages and reduces microenvironmental influences at the study site (Gove et al. 2009).

\section{New ant and plant species}

The plant community recorded for Network 2000 had a higher proportion of new plant species, reflecting the fast-paced, recent increase in agricultural and cattleraising activities that favor the presence of extensive populations of ruderal species that have tended to displace native plant species in the study site (MorenoCasasola 2006). The diversification of the ant community in Network 2000 is characterized by the presence and high number of links of five new species (McGlynn 1999, Schultz and McGlynn 2000, Holway et al. 2002, Ness and Bronstein 2004), which, besides the introduced ant Paratrechina longicornis, were absent in Network 1990.

New ants entered the community but did not alter its nested structure. Aizen et al. (2008) found in a plantpollinator network that new species integrated into native networks without influencing connectance. Our results exhibit a similar tendency because, although our more recent ant-plant network has considerably higher connectance than 10 years previous, new species were not responsible for this increase as almost all were species with few associations. Native generalists instead were responsible for this pattern. Paratrechina longicornis, the only "invasive" ant species recorded in Network 1990, was peripheral but became a core species in the more recent network. No similar case happened with plants.

The role of exotic species in network structure has been a central issue in recent analyses. Aizen et al. (2008) found that in highly invaded pollination webs, many species interacted with generalist aliens, and more species became highly dependent on them. However, we found that the new species developed few links with other species. We do not know when each appearance 
occurred but, for three new species that occurred in both networks (the ant $P$. longicornis, and the plants Crotalaria incana and Cordia dentata), the number of interactions seems to have increased with time. They had an average number of links lower than the mean in Network 1990, while 10 years later (Network 2000) they were the only species with average numbers of links larger than the mean. As suggested by Olesen et al. (2002), new species do not interact with their new counterparts as much as expected by chance. A similar change was suggested for plant-pollinator networks (Memmot and Waser 2002), where upon arrival, newcomers were rare and probably persisted in networks through interactions with native generalists, so they engaged in few interactions and exhibited limited integration due to their scarcity.

As in other mutualistic networks (Olesen et al. 2002, Aizen et al. 2008), we did not find invader complexes. We found that new species spread within the networks (Bascompte and Jordano 2007), and interacted mostly with the generalists (many of which were core species within each network) and not with their new counterparts. Our results indicate that for the ant-plant networks the core of generalists largely modulates how new species build up their interactions within the community. In contrast, Aizen et al. (2008) found that super-generalist new species in pollination networks quickly form the core of highly invaded webs. It is possible that the structure of new species in these antplant networks resembles more the "lightly invaded webs" described by Aizen et al. (2008). Another possibility is that the difference might be also related to the recurrent successional changes in our field site due to dune dynamics resetting the community to "light" invasion levels. This seems to be the case for the new ant species, which were scarcely represented in both our networks. They represented $4.3 \%$ of total ant species in Network 1990 and 16.7\% in Network 2000. It also could be the case for the new plant species in Network 1990 (representing $16 \%$ of total plant species), but unlikely for new plant species after 10 years (Network 2000) since they represented $32.5 \%$ of the total number of plants bearing extrafloral nectaries (EFNs). Aizen et al. 2008 consider highly invaded webs as those having 28\%-59\% of new plant species.

\section{Conclusion}

Here we addressed two of the so-considered "future issues" on mutualistic complex network studies (Bascompte and Jordano 2007). (1) How do mutualistic networks change in time? (2) What are the communitywide consequences of species invasions? We found that some patterns were invariant, such as nestedness, despite the incorporation of new species, while others were likely to change, such as generalization and dissimilarity. Generalist species seem to account for these patterns, since the new species incorporated within the communities are linked to the core of generalists.
Even though the results presented here are qualitative, because we did not consider the frequency of visits of an ant species to a plant species, we found significant positive relationships between species strength (frequency of an interaction in quantitative networks; Blüthgen et al. 2007) and species degree (number of links in qualitative networks), as has been reported in other studies (Vázquez et al. 2005, Bascompte et al. 2006). Thus, the distribution of links among species can show the asymmetries in specialization in these networks and suggests how the strength of the interactions may vary among particular sets of species. If asymmetry in specialization is a key factor for diversity and coexistence of species-rich communities (e.g., Bascompte et al. 2006, Bascompte and Jordano 2007), then ant-plant EFN-mediated interactions could be fundamental in maintaining diversity, not only for those groups, but also for the array of other organisms that depend on them (i.e., multitrophic interactions). Also, because many plant and ant species at the site are true mutualists that increase each other's fitness through their association (Rico-Gray and Oliveira 2007), the ongoing incorporation of ant and plant species into the more recent network (including alien species) may provide novel opportunities for the study of mutualism and coevolution (Thompson 2005, 2009, Guimarães et al. 2007). The temporal approach to the study of mutualistic networks that we have undertaken in this study provides the kinds of data needed to assess the robustness of local mutualistic networks that are increasingly faced with growing numbers of new species.

\section{ACKNOWLEDGMENTS}

R. J. Marquis thanks John Flunker, Diego Salazar, Beatriz Baker, and Scott Powell for their feedback. We thank two annonymous reviewers, whose comments and suggestions greatly improved the manuscript. Research was supported by grant 46840 from CONACYT to V. Rico-Gray, CONACYT fellowship 51721 to C. Díaz-Castelazo, and FAPESP and CAPES fellowships to P. R. Guimarães, Jr.

\section{Literature Cited}

Aizen, M. A., C. L. Morales, and J. M. Morales. 2008. Invasive mutualists erode native pollination webs. PLoS Biology 6: 396-403.

Atmar, W., and B. D. Patterson. 1993. The measure of order and disorder in the distribution of species in fragmented habitat. Oecologia 96:373-382.

Bascompte, J., and P. Jordano. 2006. The structure of plantanimal mutualistic networks. Pages 143-159 in M. Pascual and J. A. Dunne, editors. Ecological networks: linking structure to dynamics in food webs. Oxford University Press, Oxford, U.K.

Bascompte, J., and P. Jordano. 2007. Plant-animal mutualistic networks: the architecture of biodiversity. Annual Review of Ecology, Evolution and Systematics 38:567-593.

Bascompte, J., P. Jordano, C. J. Melián, and J. M. Olesen. 2003. The nested assembly of plant-animal mutualistic networks. Proceedings of the National Academy of Sciences (USA) 100:9383-9387.

Bascompte, J., P. Jordano, and J. M. Olesen. 2006. Asymmetric coevolutionary networks facilitate biodiversity maintenance. Science 312:431-433. 
Blüthgen, N., F. Menzel, T. Hovestadt, B. Fiala, and N. Blüthgen. 2007. Specialization, constraints, and conflicting interests in mutualistic networks. Current Biology 17:341346.

Borgatti, S. P., and M. G. Everett. 1999. Models of core/periphery structures. Social Networks 21:375-395.

Borgatti, S. P., M. G. Everett, and L. C. Freeman. 1999. UCINET 5.0, Version 1.00. Analytic Technologies, Natick, Massachusetts, USA.

Borgatti, S. P., M. G. Everett, and L. C. Freeman. 2002. UCINET 6 for Windows: software for social network analysis. Analytic Technologies, Harvard, Massachusetts, USA.

de Nooy, W., A. Mrvar, and V. Batagelj. 2005. Exploratory social network analysis with Pajek. Cambridge University Press, New York, New York, USA.

Díaz-Castelazo, C., V. Rico-Gray, P. S. Oliveira, and M. Cuautle. 2004. Extrafloral nectary-mediated ant-plant interactions in the coastal vegetation of Veracruz, México: richness, occurrence, seasonality and ant foraging patterns. Ecoscience 11:472-481.

Díaz-Castelazo, C., V. Rico-Gray, F. Ortega, and G. Angeles. 2005. Morphological and secretory characterization of extrafloral nectaries in plants of coastal Veracruz, Mexico. Annals of Botany 96:1175-1189.

Fonseca, C. R., and G. Ganade. 1996. Asymmetries, compartments and null interactions in an Amazonian ant-plant community. Journal of Animal Ecology 65:339-347.

Gove, A., J. Majer, and V. Rico-Gray. 2009. Ant assemblages in isolated trees are more sensitive to species loss and replacement than their woodland counterparts. Basic and Applied Ecology 10:187-195.

Guimarães, P. R., Jr., and P. Guimarães. 2006. Improving the analyses of nestedness for large sets of matrices. Environmental Modelling Software 21:1512-1513.

Guimarães, P. R., Jr., V. Rico-Gray, P. S. Oliveira, T. Izzo, S. F. Reis, and J. N. Thompson. 2007. Interaction intimacy affects structure and coevolutionary dynamics in mutualistic networks. Current Biology 17:1797-1803.

Guimarães, P. R., Jr., V. Rico-Gray, S. F. Reis, and J. N. Thompson. 2006. Asymmetries in specialization in ant-plant mutualistic networks. Proceedings of the Royal Society B 273:2041-2047.

Holway, D. A., L. Lach, A. V. Suarez, N. D. Tsutsui, and T. J. Case. 2002. The causes and consequences of ant invasions. Annual Review of Ecology and Systematics 33:181-233.

Jordano, P., J. Bascompte, and J. Olesen. 2003. Invariant properties in coevolutionary networks of plant-animal interactions. Ecology Letters 6:69-81.

McGlynn, T. P. 1999. The worldwide transfer of ants: geographical distribution and ecological invasions. Journal of Biogeography 26:535-548.
Memmot, J., and N. Waser. 2002. Integration of alien plants into a native flower-pollinator visitation web. Proceedings of the Royal Society B 269:2395-2399.

Moreno-Casasola, P., editor. 2006. Entornos Veracruzanos: la costa de La Mancha. Instituto de Ecología, A.C., Xalapa, Veracruz, México.

Ness, J. H., and J. L. Bronstein. 2004. The effects of invasive ants on prospective ant mutualists. Biological Invasions 6: $445-461$.

Olesen, J. M., J. Bascompte, H. Elberling, and P. Jordano. 2008. Temporal dynamics in a pollination network. Ecology 89:1573-1582.

Olesen, J. M., L. I. Eskildsen, and S. Venkatasamy. 2002. Invasion of pollination networks on oceanic islands: importance of invader complexes and endemic super generalists. Diversity and Distributions 8:181-192.

Rico-Gray, V. 1993. Use of plant-derived food resources by ants in the dry tropical lowlands of coastal Veracruz, Mexico. Biotropica 25:301-315.

Rico-Gray, V., and G. Castro. 1996. Effect of an ant-aphidplant interaction on the reproductive fitness of Paullinia fuscescens (Sapindaceae). Southwestern Naturalist 41:434440.

Rico-Gray, V., and P. S. Oliveira. 2007. The ecology and evolution of ant-plant interactions. University of Chicago Press, Chicago, Illinois, USA.

Schultz, T. R., and T. P. McGlynn. 2000. The interactions of ants with other organisms. Pages 35-44 in D. Agosti, J. D. Majer, L. E. Alonso, and T. R. Schultz, editors. Ants: standard methods for measuring and monitoring biodiversity. Smithsonian Institution Press, Washington, D.C., USA.

Selva, N., and M. A. Fortuna. 2007. The nested structure of a scavenger community. Proceedings of the Royal Society B 274:1101-1108.

Simberloff, D., and B. Von Holle. 1999. Positive interactions of nonindigenous species: invasional meltdown? Biological Invasions 1:21-32.

Sokal, R. R., and F. J. Rohlf. 1995. Biometry. W. H. Freeman, New York, New York, USA.

Thompson, J. N. 2005. The geographic mosaic of coevolution. University of Chicago Press, Chicago, Illinois, USA.

Thompson, J. N. 2009. The coevolving web of life. American Naturalist 173:125-140.

Vázquez, D. P., C. J. Melián, N. M. Williams, N. Blüthgen, B. R. Krasnov, and R. Poulin. 2007. Species abundance and asymmetric interaction strength in ecological networks. Oikos 116:1120-1127.

Vázquez, D. P., W. F. Morris, and P. Jordano. 2005. Interaction frequency as a surrogate for the total effect of animal mutualists on plants. Ecology Letters 8:1088-1094.

\section{APPENDIX A}

Species codes and identities (Ecological Archives E091-058-A1).

\section{APPENDIX B}

Dissimilarity dendrograms for ant species in Networks 1990 and 2000 (Ecological Archives E091-058-A2).

\section{APPENDIX C}

Dissimilarity dendrograms for plant species in Networks 1990 and 2000 (Ecological Archives E091-058-A3). 